\title{
Mixing Automated with Non-Automated Yard Traffic in Container Terminals: a Digital Transition
}

\author{
Berry Gerrits \\ Industrial Engineering and Business \\ Information Systems \\ University of Twente \\ Enschede, The Netherlands \\ b.gerrits@utwente.nl
}

\author{
Peter Schuur \\ Industrial Engineering and Business \\ Information Systems \\ University of Twente \\ Enschede, The Netherlands \\ p.c.schuur@utwente.nl \\ Sofia Kalyazina \\ Graduate School of Business and \\ Managements \\ Peter the Great St.Petersburg \\ Polytechnic University \\ Saint Petersburg, Russia \\ kaliazina.s@gmail.com
}

\author{
Igor Ilin \\ Graduate School of Business and \\ Managements \\ Peter the Great St.Petersburg \\ Polytechnic University \\ Saint Petersburg, Russia \\ ilyin.igor@eei.spbstu.ru
}

\begin{abstract}
This paper addresses how the transition of a (nonautomated) brownfield terminal to a terminal with mixed automated and manual traffic is impacting: (i) design (e.g., terminal layout planning), (ii) operational control and (iii) digital infrastructure. We propose a (digital) transition process towards coexistence of automated and manual traffic, so as to ensure efficient, secure and future-proof container handling.
\end{abstract}

Keywords - Automated Yard Tractors, Container Terminals, Multi-agent Systems, Vessel Turnaround Time, Smartification

The reported study was funded by RSCF according to the research project № 19-18-00452

\section{INTRODUCTION}

To increase productivity, container terminals worldwide adopt a broad range of automated systems, such as Automated Guided Vehicles (AGVs), automated Ship-To-Shore (STS) cranes and Automated Stacking Cranes (ASCs). Typically, in these Automated Container Terminals (ACTs), the AGVs are strictly separated from non-automated equipment or are deployed in areas where no workers or other external equipment (e.g., road trucks) are allowed. Introducing automated solutions in container terminals requires (digital) infrastructural changes. AGVs are therefore typically only deployed in newly built terminals (i.e., greenfield), whereas already existing terminals (i.e., brownfield) suffer from low adaptation rates of $\mathrm{AGVs}$ or other automated vehicles.

A commonly used vehicle in container terminals is the Yard Tractor (YT). Recent advancements in autonomous driving have created opportunities for brownfield terminals to

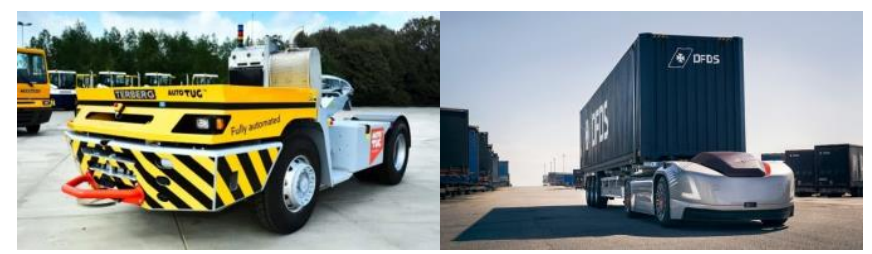

Fig. 1. Examples of Automated Yard Tractors (AYTs). introduce a new generation of these YTs as they are now able to drive autonomously and unmanned by using sensors like GPS, radars and lidars. These Automated Yard Tractors (AYTs), as exemplified in Fig. 1, also pose additional challenges. Because the AYTs drive through the stacks of a container terminal (i.e., the stacks are parallelly oriented to the quay), they also face (non-automated) road trucks, to deliver and pickup containers. This results in a mixed traffic situation which has not been addressed in the literature before, due to the novelty of the technology, as opposed to AGVs which have been extensively researched. Because current ACTs have strictly disconnected zones for automated and non-automated transport, typically separated by the stacks (i.e., perpendicularly oriented), mixed traffic was not yet considered.

Literature on ACTs is abundant and focuses on topics like routing [1-4], dispatching [5-6], deadlocks [7-8], container stacking [9-10], yard cranes [11-12] and transportation systems [13-14]. Moreover, the impact of autonomous solutions for horizontal transport (e.g., AGVs or automated straddle carriers) has been explored by many researchers. The interested reader is referred to the reviews in [15] and [16]. As pointed out by [17], it is a challenge to integrate automated and non-automated cargo handling equipment. Close to this research is the work of [18] which explores AGVs at perpendicularly oriented ACTs but does not allow for nonautomated handling equipment. Since current literature does not adequately address the impact of allowing mixed traffic in ACTs, we aim to fill this gap by exploring the potential and implications of mixing AYTs with manual yard traffic.

This paper therefore addresses how the transition of a (non-automated) brownfield terminal to a terminal with AYTs and mixed traffic is impacting (i) design (e.g., terminal layout planning), (ii) operational control and (iii) digital infrastructure. The paper proposes a (digital) transition process that ensures efficient, safe, secure and future-proof container handling. 
The remainder of this paper is structured as follows. Section 2 describes the use of AYTs in a hypothetical terminal with realistic characteristics. Section 3 describes the results of the application of AYTs and the identified difficulties that need to be addressed, Section 4 summarizes the issues identified and provides directions for future research. We close with conclusions in Section 5.

\section{CASE DESCRIPTION}

To guide the discussion on the impact of mixed traffic at ACTs using AYTs, let us focus on the hypothetical terminal shown in Fig. 2. It features four STS cranes to serve vessels and twelve stacks to temporarily store containers. Each STS has a dedicated lane for the (un)loading of containers to AYTs, as shown by the matching colors in Fig. 2. Each stack is serviced by an ASC which interacts with AYTs via a lane at either the top or bottom of the stack. We use the top six stacks to store export (or transshipment) containers and the bottom six for import containers. In this way, containers that are loaded or unloaded to and from the vessel are stored close to the waterside and containers which are further processed via road or train are stored closer to the landside. Furthermore, a commonly used routing system from practice is employed. The AYTs drive underneath the STSs and between the stacks to pick-up and drop-off containers by following the black lines. More specifically, between the stacks there are three lanes: two Pick-up and Drop-off (P/D) lanes underneath the ASCs for (un)loading and one passageway, separated by yellow markings, as shown in Fig. 3. The passageway is used to drive between the stacks. The AYTs switch to a P/D lane from the main passageway, just before the P/D location. When the (un)loading procedure is completed, the AYTs switch back to the main passageway as soon as possible. Road trucks enter the terminal via the entry point at the bottom left of Fig. 2. They follow the dotted lines through the stacks and leave the system at the bottom right. Road trucks drive to import stacks to pick-up a container and to export stacks to drop-off a container. They follow the same procedure for (un)loading at the P/D lanes as the AYTs.

Inherent to this commonly used type of terminal, is that AYTs and road trucks need to share lanes, resulting in a mixed traffic system. Current practice at terminals worldwide is to either use only non-automated vehicles (both YTs and road trucks), or when automated vehicles are used, not to mix them with other traffic. Having a driver on all vehicles simplifies traffic control and can be controlled by using simple traffic

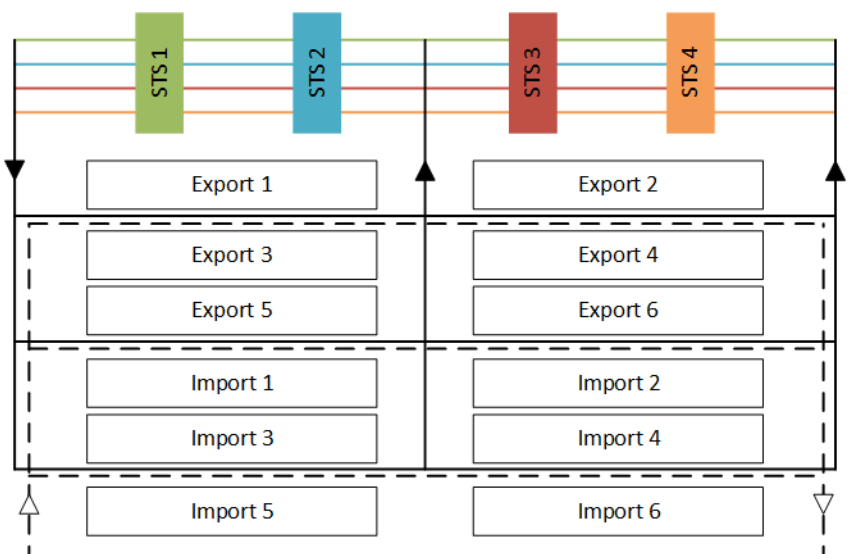

Fig. 2. Layout of the ACT under consideration. rules. However, when YTs are automated, there is no driver to take control. Comparable to currently deployed ACTs with AGVs, we propose to use a digital control system for the AYTs to replace the tasks that were previously taken care of by the driver (e.g., giving way, steering and stopping). Specifically, this control system should mitigate safety risks due to the unpredictable behavior of the external (manual)

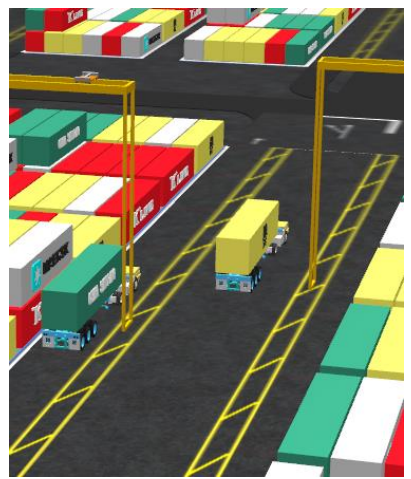

Fig. 3. Close-up of the three lanes between the stacks.

truck drivers.

\section{RESULTS}

Based on the case study, we discuss the impact of AYTs on brownfield terminals allowing for mixed traffic. The ways in which terminals are influenced by the transition to AYTs are discussed in the sections below and suggestions are made on how to manage this transition.

\section{A. Terminal layout and vehicle localization}

The layout of a terminal is essential for studying the impact of AYTs and the first place to start the analysis. As we focus on brownfield terminals, the layout and infrastructure are fixed, but terminals differ in for example dimensions (e.g., number of stacks), berth locations and hinterland connections. However, they are typically designed similarly to the layout shown in Fig. 2. To transition smoothly to AYTs, the layout and infrastructure should not be required to change when implementing AYTs. This need not be a major issue, as the layout is already adapted to (non-automated) YTs and the dynamics and kinematics of AYTs are similar. However, depending on the density and size of the terminal, GPS reception might lack in some areas and consequently might distort the (presumed) location and orientation of AYTs. In these situations, additional GPS beacons are required, or other solutions for precise location and orientation measurement need to be found. Solutions might include fixed camera systems with advanced vehicle recognition software. A similar approach, but with camera-equipped drones, might offer more flexibility over fixed cameras. Decision-makers should not underestimate the difficulty in precisely determining the orientation of an AYT as it is an articulated vehicle (i.e., an AYT with container chassis). Failure to do so may lead to miscalculated paths and therefore poor synchronization with other equipment like ASCs, deadlocks or even crashes.

\section{B. Fleet size}

As terminals worldwide face increasing pressure to turn ships around in a timely manner, terminals are increasing in size. This is illustrated by the development of the Tuas terminal in Singapore, with an expected capacity of 65 million 
TEU in 2040. These developments also require large fleets of vehicles to move containers around in order to meet performance requirements, such as the Vessel Turnaround Time (VTT). The VTT is highly determined by the utilization of the STS cranes. Hence, waiting times due to the unavailability of an AYT should be minimized. However, simply increasing the number of AYTs does not guarantee performance increase due to possible increased congestion. Furthermore, as AYTs are expected to be significantly more expensive than non-automated YTs, decision makers should carefully select the number of vehicles in the system based on the desired performance of the terminal. As manual YTs offer more flexibility than AYTs (e.g., by cutting corners, maintaining short following distances or quick acceleration) it is expected that automating YTs requires more vehicles to reach the same performance. This is mainly due to the digital safety systems required for AYTs, which are more restrictive compared to manually operated YTs.

\section{Vehicle pooling}

Typically, in terminals with manually operated YTs, the drivers are assigned to a single STS crane in order to simplify tasks. When AYTs are deployed, a fixed STS assignment is no longer required as a digital control system can easily assign different STSs to AYTs. Therefore, a full or partial pooling system can be introduced. In a full pooling system, all AYTs can serve all STSs, whereas in a partial pooling system a subset of vehicles is assigned to a subset of cranes (e.g., to prioritize a set of cranes serving a specific vessel). This increases the flexibility of the fleet and may provide a robust solution for dispatching under uncertainty.

\section{STS lane assignment}

Although mixed traffic is not applicable to the quay area, the transition from YTs to AYTs does have impact on this area. We propose to use a fixed P/D lane per STS for terminals with AYTs. Brownfield terminals with YTs may also use a fixed P/D lane per STS, but they frequently use multiple lanes per STS; whenever a lane is occupied, the YT driver will drive the vehicle around. For AYTs this requires complex maneuvering and may result in safety issues as the movements of AYTs are less predictable. Furthermore, if a terminal allows AYTs to merge to the P/D lanes between the STS cranes (i.e., the black line between STS 2 and 3 in Fig. 2), then the lane assignment is important. If such a merge is allowed, we denote this by an STS shortcut. If STS shortcuts are used, the AYT needs to check traffic coming from the right. Depending on the position of the STS crane on the right of the shortcut (i.e., STS 3 in Fig. 2), it may create a blind spot for the digital sensors of the AYT. Also, AYTs may be waiting underneath the STS on the right, further increasing the blind $\operatorname{spot}(\mathrm{s})$ of the AYT. To avoid possible collisions, the AYT would almost need to come to a full stop in order to accurately map its surroundings and detect traffic approaching from the right. This puts pressure on the vehicle detection system and increases the complexity of the vehicle detection software required for a safe and efficient traffic system.

We propose two directions to cope with this issue: (i) maintaining a specific lane assignment and (ii) to deny access between the STS cranes. The latter completely removes the STS shortcut(s) and implies that AYTs may not enter the STS area before the rightmost STS serving a vessel. If we assume that AYTs are randomly assigned to STS cranes (i.e., full pooling) and to the stacks, $50 \%$ of the AYTs face approximately $50 \%$ more travel distance for 2 out of the 4 STSs, resulting in approximately $12.5 \%$ more travel distance. The impact of this increase on the VTT should be further researched, for example by using simulation. Another solution is to assign P/D lanes to STSs such that there are no possible conflicts with traffic approaching from the right. This is accomplished by simply assigning the lanes from top to bottom to the STSs from right to left. This is the exact reverse allocation from that shown in Fig. 2.

\section{E. Dynamic traffic rules}

Deploying AYTs and digital control systems allows for non-traditional traffic rules. While traditional traffic rules may be adopted to ensure an understandable and predictable traffic system, exceptions can be made based on the dynamic state of the terminal. For example, (certain) AYTs may be given way when the VTT drops, or road trucks may be preferred over AYTs at crossings when queues start to clog the system. When non-traditional traffic rules are employed, sharing information on them with road trucks is crucial for safety. Traffic lights or Variable Message Signs (VMSs) play a key role in the transition to dynamic traffic rules. Practitioners should balance the potential increase in operational performance due to dynamic traffic rules with the understandability of the rules for the non-automated part of the mixed traffic.

\section{F. Sensor requirements and lane changes}

Crucial for digital control of AYTs are on-board sensors as well as communication technology. The AYTs communicate with each other and exchange information about their own status as well as traffic around them (e.g. obstacles). Essential is the communication with the manual drivers. The control system should issue alerts to them (e.g. on accidents, detours, speed recommendations, etc.) by means of VMSs [19]. On-board sensors should be able to accurately measure relative distance, angle, and speed of the AYT compared to all nearby vehicles. Forward looking longitudinal sensors must have sufficient range to allow collision-free emergency stops. Backward looking sensors must detect potential rear-end collision threats. They are also needed to determine the available spacing during lane changing. Lateral sensors also serve the latter purpose. They detect if there is any vehicle in the destination lane or coming towards it.

\section{G. Speed harmonization}

Container terminals need to adopt clear regulations and traffic rules, taking into account AYTs and road trucks simultaneously. It is also necessary to harmonize speeds between AYTs and road trucks. This provides a compromise between compliance with safety requirements, efficient operations and the optimal number of vehicles used. As observed by [19] introducing automated vehicles in mixed traffic may dampen speed oscillations as these vehicles have restricted acceleration and deceleration. This may result in a more harmonious traffic flow and will keep manual drivers from sudden speed changes.

In our case, AYTs may be restricted to a speed of no more than $20 \mathrm{~km} / \mathrm{h}$ and road trucks should also adhere to this speed limit to create a safe traffic system. Even though the theoretical maximum speed of AYTs may be well above this speed limit, a trade-off should be made between safety (i.e., speed harmonization) and operational performance, in terms 
of VTT. Contrary to [19], traffic in our case is restricted to a secluded area, allowing for a forest of road sensors with which the manual driver can be monitored. Speed violation is detected immediately and may lead to appropriate interventions. Furthermore, due to the digital control of AYTs, speed limits may be dynamically allocated throughout the day, depending on the state of the system (e.g., decrease the speed limit during rush hours). As discussed under Section F, these dynamic speed limits may be communicated to manual drivers by Variable Message Signs.

\section{H. Digital control system hierarchy}

As stated in Section B, the fleet of vehicles required to turn vessels around in a timely manner is expected to grow. Apart from causing congestion, deadlock and traffic control issues, it also puts pressure on the (vehicle) control systems required for effective fleet management. To cope with these challenges, we propose a transformation from centrally oriented control systems to decentralized control systems for AYTs. Currently, in many (semi-)automated and non-automated terminals, centralized control systems are used and typically resort to global optimization methods to plan and control the transportation resources, e.g., AGVs or YTs. However, these methods may be less suitable for real-time decision making in large-scale, stochastic and dynamic environments, since they typically (i) require a lot of information in advance, (ii) are sensitive to information updates, (iii) are not able to respond in a timely manner, and (iv) are not flexible enough to deal with dynamic environments and situations with multiple autonomous actors [20].

We therefore propose to use decentralized control systems, where the AYTs independently control their own driving by using advanced sensor systems to detect and map their surroundings. An additional benefit of this approach is that few infrastructural changes are required (e.g., no transponders are required, as typically used with AGV systems). Note that a mixed form of central and decentral systems might also be suitable, where for example the dispatching of AYTs is done centrally, and the actual vehicle control decentrally. Before implementing AYT systems, decision makers need to be aware of the characteristics of the various types of hierarchal control in terms of efficiency, scalability and robustness.

\section{Exploiting related trends}

The use of AYTs requires the consideration of a large number of factors, both in terms of safety as well as design characteristics. Digitalization of the container handling process by using AYTs, may benefit from exploiting findings in other areas of research. For instance, in order to meet the strict service requirements of a terminal, we may use adaptive, self-organizing systems based on multi-agent technology, thereby combining insights from object-oriented programming, artificial intelligence, telecommunication and parallel computing. Such systems are able to demonstrate the phenomena of swarm intelligence and more complex collective intelligence, which are helpful in managing a mixed traffic container terminal. As AYTs and terminals are equipped with many sensors and digital devices, we may benefit from Internet of Things (IoT) technology [21]. Moreover, the ability to adapt in real time by using an agentbased approach, improves the efficiency of resources. Making decisions in real time is accompanied by the identification and resolution of conflicts between agents with systems that have numerous individual preferences and limitations. The speed of decision making within such systems exceeds the capabilities of a human [22]. Furthermore, agents are able to respond to changes in their environment (e.g., through data-processing of the sensors of the AYTs) and are thus also adept to cope with (unexpected) behavior of road trucks. As we allow for mixedtraffic, practitioners also need to pay attention to humanmachine interaction and the human factors associated with introducing AYTs to ensure sustainable coexistence of both automated and non-automated vehicles.

\section{DISCUSSION}

Traffic automation is unstoppable. With its undeniable benefits an army of challenges marches towards us. How to enforce peaceful coexistence of automated and manual traffic? The safe, secluded area of a container yard offers a manifold of options to serve as a playing ground. Joint smartification is the keyword: it holds for automated yard traffic as well as for the yard itself. Together they have to face their natural enemy: the unpredictable, manual road truck. Future technology will lead the way. First at the yard. Eventually at the highway. Further research is needed to develop models for the optimal design and management of the equipment involved. In particular, it is key to develop a rulebased system that guarantees safe traffic in the container terminal.

\section{CONCLUSIONS}

This paper discusses the transition of a (non-automated) brownfield terminal towards a terminal that mixes Automated Yard Tractors (AYTs) with (manual) road trucks. We consider the potential benefits of introducing AYTs versus the difficulties that mixing brings. To deal with the latter, we propose a (digital) transition process towards a sensor-based, self-organizing multi-agent system ensuring efficient, secure and future-proof container handling.

\section{REFERENCES}

[1] R. Stahlbock and S. Vo $\beta$, "Vehicle Routing Problems and Container Terminal Operations - An update of Research," in The Vehicle Routing Problem: Latest Advances and New Challenges, ORCS, vol. 43, B. Golden, S. Raghavan and E. Wasil, Eds. Springer Science, 2008, pp. 551-589.

[2] E. Gawrilow, E. Köhler, R.H. Möhring, and B. Stenzel, "Dynamic Routing of Automated Guided Vehicles in Real-Time," in Mathematics Key Technology for the Future, H.J. Krebs and W. Jäger, Eds. Springer, Berlin, Heidelberg, 2008, pp. 165-177.

[3] L. Qio, and W-J. Hsu, "A bi-directional path layout for conflict-free routing of AGVs," International Journal of Production Research, 39:10, 2001, pp. 2177-2195.

[4] H. Fazlollahtabar, and M. Saidi-Mehrabad, "Methodologies to Optimize Automated Guided Vehicle Scheduling and Routing Problems: A Review Study," Journal of Intelligent \& Robotic Systems, Vol. 77(3-4), March 2015, pp. 525-545.

[5] K.H. Kim, and J.W. Bae, "A Look-Ahead Dispatching Method for Automated Guided Vehicles in Automated Port Container Terminals," Transportation Science, Vol. 38(2), 2004, pp. 224-234.

[6] M. Grunow, H-O. Günther, and M. Lehmann, "Dispatching multi-load AGVs in highly automated seaport container terminals," OR Spectrum, Vol. 26, 2004, pp. 211-235.

[7] K.H. Kim, S.M. Jeon, and K.R. Rye, "Deadlock prevention for automated guided vehicles in automated container terminals," OR Spectrum, Vol. 28(4), 2006, pp. 659-679.

[8] M. Lehmann, M. Grunow, and H-O. Günther, "Deadlock handling for real-time control of AGVs at automated container terminals," OR Spectrum, Vol. 28(4), 2006, pp. 631-657. 
[9] R. Dekker, P. Voogd, and E. van Asperen, "Advanced method for container stacking," in Container Terminals and Cargo Systems: Design, Operations Managements, and Logistics Control Issues, K.H. Kim and H-O Günther, Eds. Springer, Berlin, Heidelberg, 2007, pp. 131-154.

[10] T. Park, R. Choe, Y. Hun Kim, and K. Ryel Ryu, "Dynamic adjustment of container stacking policy in automated container terminal," International Journal of Production Economics, Vol. 133(1), 2011, pp. 385-392.

[11] A.H. Gharehgozli, G. Laporte, Y. Yu, and R. de Koster, "Scheduling twin yard cranes in a container blocks," Transportation Science, Vol. 49(3), 2015, pp. 686-705.

[12] N. Kemme, "Effects of storage block layout and automated yard crane systems on the performance of seaport container terminals," OR Spectrum, Vol. 34(3), 2012, pp. 563-591.

[13] I.F.A. Vis, and I. Harika, "Comparison of vehicle types at an automated container terminal," OR Spectrum, Vol. 26(1), 2004, pp. 117-143.

[14] M.B. Duinkerken, R. Dekker, S.T.G.L., Kurstjens, J.A. Ottjes, N.P. Dellaert, "Comparing transportation systems for inter-terminal transport at the Maasvlakte container terminals," OR Spectrum, Vol. 28(4), 2006, pp. 469-493.

[15] I.F.A. Vis, "Survey of research in the design and control of automated guided vehicle systems," European Journal of Operational Research, Vol. 170(3), 2006, pp. 677-709.
[16] E. Kaoud, M.A. El-Sharief, and M.G. El-Sebaie, "Scheduling problems of automated guided vehicles in job shop, flow shop and container terminal, " $4^{\text {th }}$ International Conference on Industrial Engineering and Applications, 2017, pp. 60-65.

[17] K. Mcginley, "Preparing port container terminals for the future: making the most of Intelligent Transport Systems (ITS)," in Urban Transport XX, C.A. Brebbia, Eds. WITpress, 2014, pp. 419-427.

[18] B. Gerrits, M. Mes, and P. Schuur, "A simulation model for the planning and control of AGVs at automated container terminals," In Proceedings of the 2018 Winter Simulation Conference, 2019, pp. 2941-2952.

[19] P. Loannou, A. Bose. "Evaluation of mixed automated/manual traffic," USC CATT Report No. 97-09-10, September 1997.

[20] M. Mes, M. Van Der Heijden, A Van Harten. "Comparison of agentbased scheduling to look-ahead heuristics for real-time transportation problems," European journal of operational research, 2007, pp. 59-75.

[21] A.I. Levina, A.S. Dubgorn, O.Y. Iliashenko "Internet of things within the service architecture of intelligent transport systems" Proceedings 2017 European Conference on Electrical Engineering and Computer Science, EECS 2017, pp. 351-355.

[22] G. Rzevski, P. Skobelev "Managing complexity” Wit Press, 2014. 